\title{
KKMC-hh for Precision Electroweak Phenomenology at the LHC
}

\author{
Scott A. Yost, ${ }^{a, *}$ M. Dittrich, ${ }^{a}$ S. Jadach, ${ }^{b}$ B.F.L. Ward ${ }^{c}$ and Z. Wąs ${ }^{b}$ \\ ${ }^{a}$ The Citadel, \\ 171 Moultrie St., Charleston, SC 29409, USA \\ ${ }^{b}$ Institute of Nuclear Physics, Polish Academy of Sciences, \\ ul. Radzikowskiego 152, 31-342 Kraków, Poland \\ ${ }^{c}$ Baylor University, \\ Waco, TX 75798, USA \\ E-mail: scott.yost@citadel.edu, mdittric@citadel.edu, \\ stanislaw.jadach@cern.ch, bfl_ward@baylor.edu, z.was@cern.ch
}

We describe the program KKMC-hh, which calculates $\mathrm{Z}$ boson processes in hadronic collisions using coherent exclusive exponentiation (CEEX) with exact second-order photonic corrections at next-to-leading log and first-order weak vertex corrections, including initial and final state photonic radiation and initial-final interference. We describe current applications to precision forward-backward asymmetry calculations for the measurement of the electroweak mixing angle at the LHC.

40th International Conference on High Energy physics - ICHEP2020

July 28 - August 6, 2020

Prague, Czech Republic (virtual meeting)

\footnotetext{
${ }^{*}$ Speaker
} 


\section{Introduction and Program Structure}

KKMC-hh[1] is a precision MC generator for Z production and decay in high-energy proton collisions. It is based on $\mathrm{KKMC}$, [2] which was originally developed for precision $\mathrm{Z}$ boson phenomenology in $e^{+} e^{-}$collisions, including exponentiated multiple photon effects: $e^{+} e^{-} \rightarrow Z \rightarrow f \bar{f}+n \gamma$ including exact $O(\alpha)$ and $O\left(\alpha^{2} L\right)$ initial-state radiation (ISR), final-state radiation (FSR), and initial-final interference (IFI). ( $L$ is an appropriate "big logarithm" for the process, $\ln \left(p^{2} / \mathrm{m}^{2}\right)$ for a relevant mass.) Order $\alpha$ electro-weak matrix element corrections are included via an independent DIZET module, originally version 6.21[3], but recently upgraded to version $6.45[4,5]$. Collision energies up to $1 \mathrm{TeV}$ are supported. The LEP2 precision tag was $0.2 \%$. Beginning with version 4.22, KKMC also includes support for parton-level collisions of quarks.[6]

An adaptive MC, FOAM,[7] underlies the low-level event generation. The FOAM grid is set up during an initial exploratory phase, creating a crude MC distribution that includes the PDF factors and a crude YFS form-factor for the ISR photon radiation.

KKMC generates multiple-photon radiation using one of two modes of resummation. EEX mode (exclusive exponentiation) is based on YFS soft photon resummation,[8] implemented at the cross-section level, while CEEX mode (coherent exclusive exponentiation)[9] is an amplitude-level adaptation of YFS resummation. IFI enters naturally when an amplitude including exponentiated ISR and FSR factors is squared. See Ref. [10] for a recent study of IFI in the CEEX framework.

The events generated by KKMC-hh may be showered externally by exporting them in an LHE-format event file,[11] or by running a built-in HERWIG6.5[12] shower.

\section{Photonic Radiative Corrections}

KKMC-hh takes an ab-initio calculation of photon ISR at the Feynman diagram level, with exponentiation. This is in contrast to a more traditional approach of factorizing the collinear ISR into the parton distribution functions.

A QED-corrected PDF can account for the shift in quark moment due to photonic ISR to the extent that the observable is sufficiently inclusive to average over any transverse momentum. In particular, it is reasonable to expect that KKMC-hh should show good agreement with a QEDcorrected PDF for distributions such as the invariant-mass distribution of the final leptons, in the absence of individual lepton cuts. However, KKMC-hh can go beyond the PDF approximation and account for the transverse momentum effects from the ISR that would be missed in a strictly collinear representation that effectively confines the ISR to the protons.

Fig. 1(a) shows the ratio of the invariant mass $\left(M_{l l}\right)$ distribution with ISR turned on in KKMC-hh to the distribution with ISR off (red), compared the same ratio calculated with ISR off in KKMC-hh, but using the LuxQED version of NNPDF3.1 instead of the standard version. The results are from a $10^{9}$ event muon sample generated without a QCD shower or FSR and with no cuts on the muon momenta, using current quark masses from the PDG,[13] for $8 \mathrm{TeV}$ proton $\mathrm{CM}$ energy.

Both methods of accounting for ISR lead to a shift of about $-0.5 \%$, and the distributions largely overlap to the statistical limits of the sample, suggesting a high degree of consistency between the two approaches in a case where agreement is expected. The shift in the total cross 
section for $60 \mathrm{GeV}<M_{l l}<120 \mathrm{GeV}$ was $-0.524 \pm 0.004 \%$ when turning on ISR in KKMC-hh, and $-0.624 \pm 0.002 \%$ when switching to the NNPDF3.1-LuxQED, so the two ways of accounting for ISR give cross sections in agreement to $0.1 \%$. The shift in the average $M_{l l}$ was $-0.10 \pm 0.27 \mathrm{MeV}$ when turning on ISR in KKMC-hh, and $-0.40 \pm 0.27 \mathrm{MeV}$ when switching to a LuxQED PDF instead.

Fig. 1(b) shows a similar comparison for the rapidity distribution $Y_{l l}$ for the same events. Both KKMC and the LuxQED version of NNPDF3.1 lead to a shift in the rapidity distribution on the order of $-0.5 \%$, but the shape is somewhat different, crossing at $Y=2.5$.
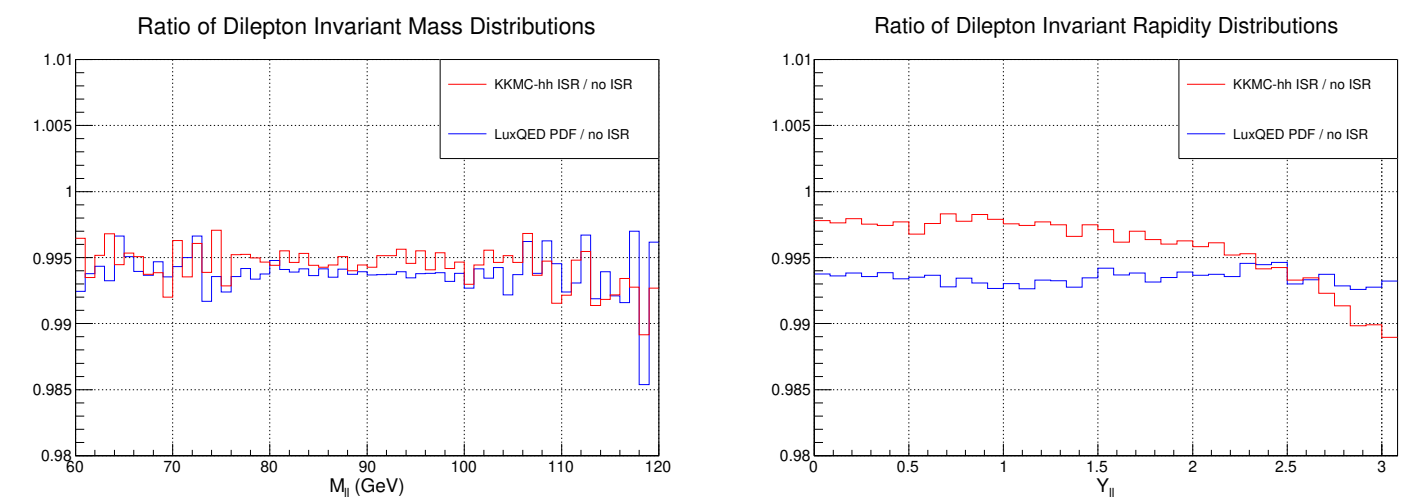

Figure 1: Comparison of the ratios of the invariant mass $\left(M_{l l}\right)$ and rapidity $\left(Y_{l l}\right)$ distributions for the final lepton pair with and without ISR corrections added using KKMC-hh (red) or switching to the LuxQED version of NNPDF3.1 (blue).

Angular distributions are of particular interest in the context of the measurement of the electroweak mixing angle at the LHC, in which KKMC-hh is participating together with other programs combining hadronic and electroweak effects, including POWHEG-EW[15], MC-SANC[16], ZGRAD2[17], and HORACE[18]. The Collins-Soper angle[19] is the scattering angle in the CM frame of the final lepton pair, given by

$$
\cos \left(\theta_{\mathrm{CS}}\right)=\operatorname{sgn}\left(P_{l l}^{z}\right) \frac{p_{l}^{+} p_{\bar{l}}^{-}-p_{l}^{-} p_{\bar{l}}^{+}}{\sqrt{P_{l l}^{2} P_{l l}^{+} P_{l l}^{-}}}
$$

neglecting masses, with $P_{l l}=p_{l}+p_{\bar{l}}$ and $p^{ \pm}=p^{0} \pm p^{z}$. The initial-final interference contribution is of particular interest for the angular distribution, since it is strongly dependent on the scattering angle.

Fig. 2 shows the CS angle distribution generated in a KKMC-hh run with $9 \times 10^{9}$ muon events at an $8 \mathrm{TeV}$ CM energy, using NNPDF3.1 PDFs[20], and without additional fermion cuts. Similar results are described in detail in Ref.[21]. The graph on the right of shows the $\cos \left(\theta_{\mathrm{CS}}\right)$ full KKMC-hh distribution (green) together with a version with IFI off (red), a version with both ISR and IFI off (black), and a version with IFI off, but ISR effects included by using a LuxQED[22] version of NNPDF3.1 instead, NNPDF3.1-LuxQED[23] (blue). The shift due to ISR is $-0.4 \%$ for both ways of accounting for ISR, to within $\pm 0.1 \%$. The IFI correction is less than $10^{-4}$, but this effect increases for less inclusive cuts, as in the binned results for the forward-backward asymmetry shown below. 

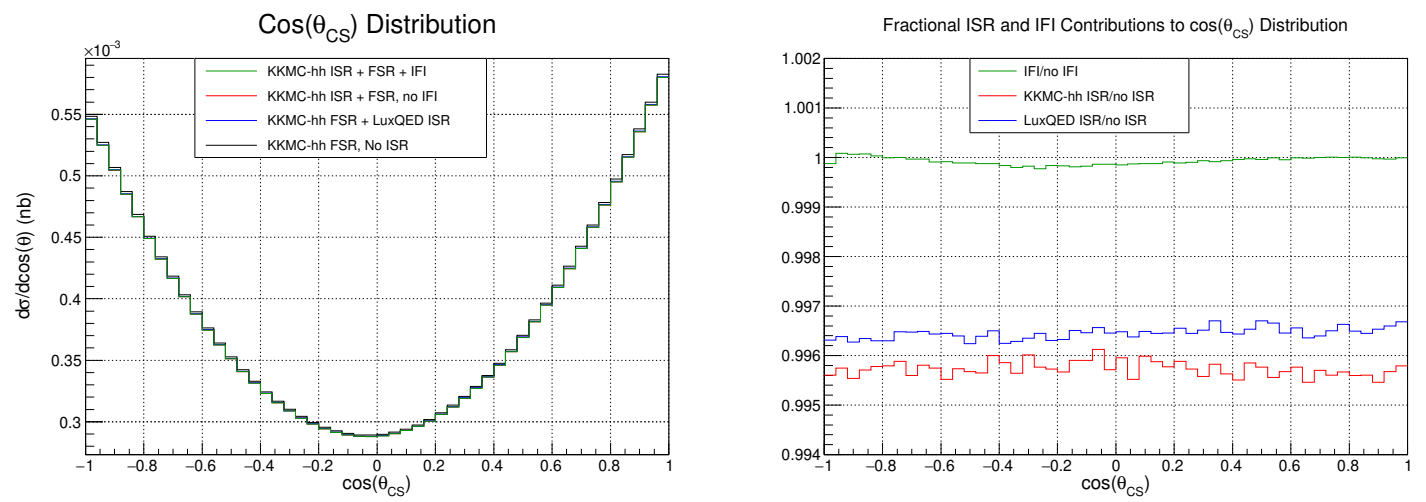

Figure 2: The Collins-Soper angle distribution with full KKMC-hh (green) compared to versions without IFI (red), without IFI and ISR (black), and without IFI but including ISR effects via a LuxQED version of the PDFs (blue). The graph on the right shows ratios with respect to a baseline result including only FSR corrections.

The effect of the photonic corrections on the forward-backward asymmetry $A_{\mathrm{FB}}$ is shown in Fig. 3 as a function of $M_{l l}$ and Fig. 4 as a function of $Y_{l l}$. The IFI effect becomes much more pronounced in these binned results, while ISR is less significant, at least for cuts close to $M_{l l}=M_{Z}$. The right-hand graph of Fig. 3 shows that IFI has an effect (green) on the order of $0.1 \%$ with a strong dependence on $M_{l l}$ in the vicinity of $M_{l l}=M_{Z}$. The KKMC-hh ISR effect is near zero and flat in the vicinity of $M_{Z}$, but with large statistical errors for larger $M_{l l}$. The ISR effect obtained by switching from NNPDF3.1 to NNPDF3.1-LuxQED, however, shows a contribution small at $M_{Z}$ but larger elsewhere, and with a pronounced slope. ISR has negligible effect in Fig. 4, but IFI is strongly enhanced at high rapidities.
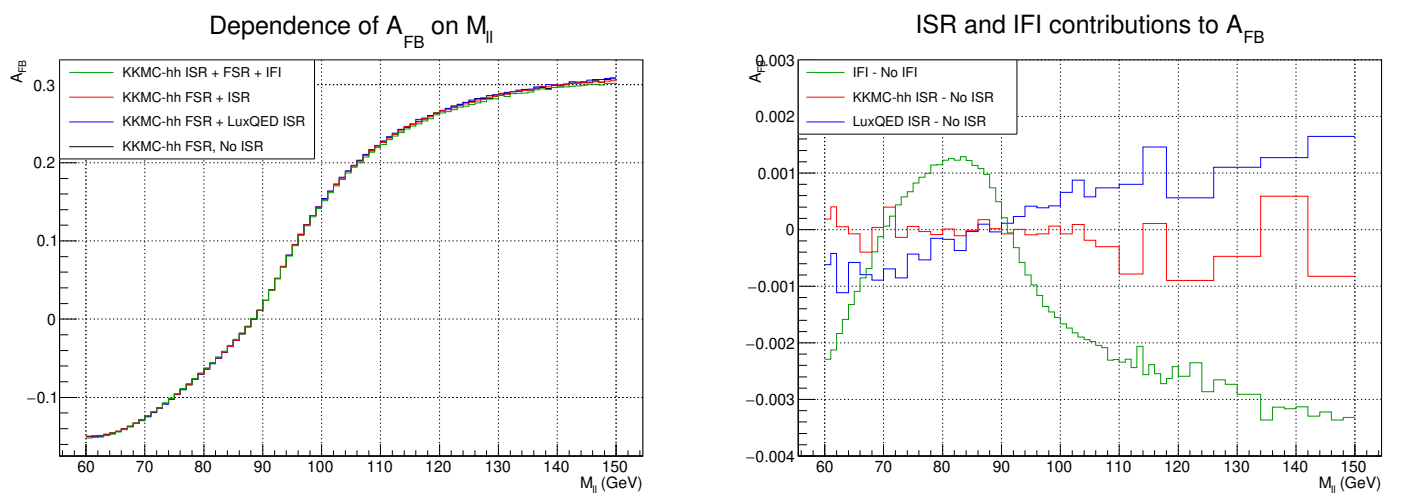

Figure 3: The forward-backward asymmetry $A_{\mathrm{FB}}$ for full KKMC-hh (green), as a function of dilepton invariant mass $M_{l l}$, compared to versions without IFI (red), without IFI and ISR (black), and without IFI but including ISR effects via a LuxQED version of the PDFs (blue). The graph on the right shows differences with respect to a baseline calculation with only FSR photonic corrections.

\section{Summary and Outlook}

KKMC-hh provides a precise tool for calculating exponentiated photonic corrections to hadron 

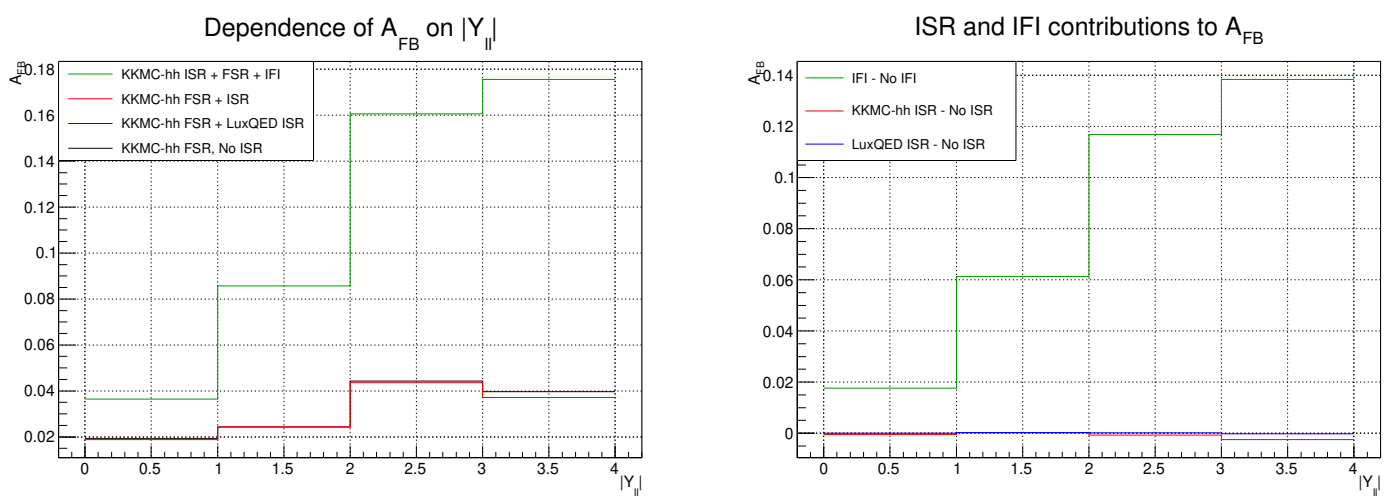

Figure 4: The forward-backward asymmetry $A_{\mathrm{FB}}$ for full KKMC-hh (green) as a function of dilepton rapidity $Y_{l l}$, compared to versions without IFI (red), without IFI and ISR (black), and without IFI but including ISR effects via a LuxQED version of the PDFs (blue). The graph on the right shows differences with respect to a baseline calculation with only FSR photonic corrections.

scattering. In particular, it can calculate contributions of ISR and IFI to the forward-backward asymmetry, which will be useful for determining the electroweak mixing angle from LHC data. KKMC-hh is particularly well suited to evaluating IFI due to its CEEX exponentiation.

While showered results were not presented in this note, they are possible both with an internal HERWIG6.5 shower, and by exporting events and applying an external shower. This will allow addressing NLO QCD effects as well. There has also been progress on a version of KKMC-hh which can be run to add electroweak corrections to previously-generated hadronic events. KKMC-hh is presently being transcoded entirely to $\mathrm{C}++$. This will facilitate compiling it with a current hadronic generator such as Herwig 7[24] or KrkNLO[25].

\section{Acknowledgments}

S. Yost acknowledges support of The Citadel Foundation and the Institute of Nuclear Physics, IFJ-PAN, Kraków, Poland, which provided computing resources for this project. S. Jadach acknowledges support of National Science Centre, Poland Grant No. 2019/34/E/ST2/00457. Z. Wa̧s was supported in part by of Polish National Science Centre under decisions DEC-2017/27/B/ST2/01391.

\section{References}

[1] S. Jadach, B.F.L. Ward, Z. Wạs, and S.A. Yost, Phys. Rev. D94 (2016) 074006 [arXiv:1608.01260].

[2] S. Jadach, B.F.L. Ward, and Z. Wạs, Comput. Phys. Commun. 130 (2000) 260 [hep$\mathrm{ph} / 9912214]$.

[3] D. Bardin, et al., Comput. Phys. Commun. 59 (1990) 303, ibid., Comput. Phys. Commun. 133 (2001) 229 [hep-ph/9908433].

[4] A.B. Arbuzov, et al., Comput. Phys. Commun. 174 (2006) 728 [hep-ph/0507146]. 
[5] A.B. Arbuzov, et al., Comput. Phys. Commun. 260 (2021) 107734

[6] S. Jadach, B.F.L. Ward, and Z. Wạs, Phys. Rev. D88 (2013) 114022 [arXiv:1307.4037].

[7] S. Jadach, Comput. Phys. Commun. 130 (2000) 244 [arXiv:physics/99100004]; ibid., Comput. Phys. Commun. 152 (2003) 55 [arXiv:physics/0203033].

[8] D.R. Yennie, S.C. Frautschi and H. Suura, Ann. Phys. 13 (1961) 379; K.T. Mahanthappa, Phys. Rev. 126 (1962) 329.

[9] S. Jadach, B.F.L. Ward, and Z. Wa̧s, Phys. Lett. B449 (1999) 97 [hep-ph/9905453], ibid., Phys. Rev. D63 (2001) 113009 [hep-ph/0006359].

[10] S. Jadach and S.A. Yost, Phys. Rev. D100 (2019) 013002 [arXiv:1801.08611].

[11] J. Alwall, et al., Comput. Phys. Commun. 176 (2007) 300 [hep-ph/0609017]

[12] G. Marchesini, et al., Comput. Phys. Commun. 67 (1992) 465; G. Corcella, et al., JHEP 0101 (2001) 010; S. Gieseke, et al., JHEP 0402 (2004) 005.

[13] Particle Data Group, Phys. Rev. D98 (2018) 030001.

[14] S. Jadach, B.F.L. Ward, Z. Wa̧s, and S.A. Yost, Phys. Rev. D99 (2019) 076016 [arXiv:1707.06502].

[15] L. Barzé, G. Montagna, et al., Eur. Phys. J. C73 (2013) 2474 [arXiv:1302.4606].

[16] S.G. Bondarenko and A.A. Sapronov, Comput. Phys. Commun. 184 (2013) 2343 [arXiv:1301.3687]; A. Arbuzov, et al., JETP Lett. 103 (2016) no. 2, 131 [arXiv:1509.03052].

[17] U. Baur, et al., Phys. Rev. D65 (2002) 033007 [arXiv:hep-ph/0108274].

[18] C.M. Carloni Calame,et al, Phys. Rev. D69 (2004) 037301; ibid., JHEP 0505 (2005) 019; ibid., JHEP 0612 (2006) 016; ibid., JHEP 10 (2007) 109.

[19] J.C. Collins and D.E. Soper, Phys. Rev. D16 (1977) 2219.

[20] R.D. Ball, Bertone, V., Carrazza, N.P, et al., Eur. Phys. J. C77 (2017) 77:663 [arXiv:1706.00428].

[21] S. Jadach, B.F.L. Ward, Z.A. Wąs, and S.A. Yost, arXiv:2002.11692; S. Yost, S. Jadach, B.F.L. Ward and Z. Wạs, PoS (RADCOR2019) 085 [arXiv:2002.12477].

[22] A. Manohar, P. Nason, G.P. Salam and G. Zanderighi, Phys. Rev. Lett. 117 (2016) 242002 [arXiv:1607.04266]; ibid., JHEP 12 (2017) 046 [arXiv:1708.01256].

[23] V. Bertone, S. Carrazza, N.P. Hartland and J. Rojo, SciPost Phys. 5 (2018) 008 [arXiv:1712.07053].

[24] Johannes Bellm, et al., Eur. Phys. J. C76 (2016) 4, 196.

[25] S. Jadach, et al., Phys. Rev. D87 034029 [arXiv:1103.5015]; ibid., Acta Phys. Polon. B46 (2015) 2089; ibid., JHEP 10 (2015) 052 [arXiv:1503.06849]. 